\title{
La regulación política de la familia. Ideología, desigualdad y género en el Plan Integral de Apoyo a la Familia
}

\author{
Dolors COMAS-D'ARgEMIR \\ Universitat Rovira i Virgili \\ dolors.comasdargemir@urv.cat
}

\author{
Diana MARRE \\ Universitat Autònoma de Barcelona \\ diana.marre@uab.cat
}

Beatriz SAN RomÁn

Universitat Rovira i Virgili

beatriz.sanroman@urv.cat

Recibido: 19-04-2015

Aceptado: 07-04-2016

\section{Resumen}

La familia es la institución privada por excelencia y, sin embargo, es objeto de intervención política. En este artículo reflexionamos acerca de los componentes ideológicos que subyacen en las políticas públicas que afectan a la familia y el significado que tienen en el contexto en que se activan. Para ello, analizamos el Plan Integral de Apoyo a la Familia 2015-2017, elaborado por el gobierno español. En la actual fase de reactivación de las políticas neoliberales, se pretende que la familia fortalezca sus funciones de protección y cuidado de sus miembros, en correspondencia con la progresiva disminución de las responsabilidades públicas por parte del Estado. En este contexto, la protección de la maternidad resulta fundamental, pues focaliza el papel de las mujeres como madres y cuidadoras. Los cambios en la agenda de la familia se corresponden, pues, con cambios en la agenda de igualdad..

Palabras clave: Familia; mujeres; género; desigualdad social; políticas públicas, políticas familiares, biopolítica, cambios familiares.

\section{The Political Regulation of Families: Ideology, Inequality and Gender in the Integral Plan of Support to the Family}

\begin{abstract}
Family is the private institution par excellence; however, it is subject to political intervention. In this article, we reflect on the ideological components that underlie public policies affecting the family and what they mean in the context in which they are activated. We analyze the Plan Integral de Apoyo a la Familia 20152017 , drawn up by the Spanish government. In the current phase of reactivation of neoliberal policies, the family is intended to strengthen its functions of protection and care of its members, in line with the gradual reduction of public responsibilities by the state. In this context, maternity protection is essential, since it
\end{abstract}


focuses the role of women as mothers and caregivers. Thus, changes in the agenda of the family correspond to changes in the equality agenda.

Key words: Family; women; gender; inequality; public policies; family policy; biopolitics; family changes.

\section{Referencia normalizada}

Comás D`Argemir, D., D. Marre y B. San Román (2016): “La regulación política de la familia. Ideología, desigualdad y género en el Plan Integral de Apoyo a la Familia”, Política y Sociedad, 53 (3), pp. 853-877.

Sumario: Introducción, 1. Ideología, desigualdad y género en las políticas familiares en España, 2. Metodología, 3. El Plan Integral de Apoyo a las Familias 2015-2017 en el contexto de las políticas familiares, 4. Objetivos y líneas estratégicas del PIAF 2015-2017, 5. Conclusiones, 6. Documentos, 7. Bibliografía.

\section{Introducción}

Este artículo se centra en el análisis de los objetivos y estrategias detallados en el Plan Integral de Apoyo a la Familia 2015-2017 (en adelante PIAF 2015-2017), en tanto que ejemplo y caso de estudio sobre como los poderes públicos regulan la familia a través de los derechos y obligaciones atribuidos a sus miembros y la definición de los servicios y prestaciones que orientan y condicionan las estrategias y comportamientos familiares. El PIAF 2015-2017 fue aprobado en mayo del 2015 por el gobierno del Partido Popular (PP), luego de varios años de crisis económica, fuertes recortes del gasto público, y diseño y aplicación de políticas de corte neoliberal que han optado por la expansión del mercado y la reducción del papel del Estado.

La regulación política de la familia no solo se hace desde disposiciones legales relacionadas con el derecho civil, sino también desde la política social (Commaille y Martín, 1998). Donzelot ([1977] 1979) denominó como la "policía de las familias" al conjunto de tecnologías políticas de regulación que actúan desde el siglo XVIII en los países europeos como "métodos de desarrollo de la calidad de la población y del poder de la nación [para] asegurar el bienestar del Estado mediante la sabiduría de sus reglamentos y aumentar sus fuerzas tanto como sea capaz. La ciencia de la policía consiste, pues, en regular todo lo que se relaciona con el estado actual de la sociedad" (Donzelot, ([1977] 1979: 10). Las políticas familiares son, en este sentido, un dispositivo del biopoder que controla y regula la población modelando y naturalizando ciertas subjetividades (como la de la mujer-madre) (Foucault [1976] 2005). Como subrayara Ojakangas (2007), en las sociedades modernas, el poder no se ejerce por la ley y la violencia sino mediante dispositivos que interpretan la vida -o la construyen de una determinada manera- para normalizar y controlar los sujetos y los cuerpos. De esta forma, modelan el comportamiento de los individuos a través de sus creencias, intereses y deseos (Dean, 1999). El PIAF 2015-2017 muestra claramente como la familia es intervenida -"gobernada", "regulada" o "disciplinada"- desde el poder político, fijando los derechos y obligaciones de sus miembros y proveyendo un conjunto de prestaciones 
y servicios que orientan las actitudes, comportamientos y estrategias de los actores familiares.

Desde la perspectiva foucaultiana, el poder neoliberal ya no se basa en el respeto de la libertad individual y la regulación del mercado, sino que es el mercado mismo el principio que regula y organiza la labor del estado. El individuo deviene entonces "un ser comportamentalmente manipulable" (Lemke, 2001) desde mecanismos profundos y difusos basados en valores culturales presentes en la sociedad. Individuos, familias y mercado adquieren el máximo protagonismo, mientras que el Estado se reserva un papel proveedor complementario, aunque de regulación y gobernabilidad absoluto.

En el caso de España, la alternancia en el poder entre partidos conservadores y de izquierdas se ha traducido en cambios en la regulación y focalización de las políticas que inciden en la familia. Es importante tener en cuenta la ideología del partido que gobierna pues impregna el carácter de las políticas públicas que se promueven. Para los conservadores, la familia es la institución social básica a proteger, sin cuestionar los roles tradicionales de género. Para los partidos de izquierdas, el énfasis recae en los derechos individuales, en apoyar las responsabilidades familiares (no a la familia) y en las políticas de igualdad (aunque, como veremos más adelante, las políticas familiares se acaban asumiendo también como tales). En este sentido, el PIAF 20152017 es la expresión de un gobierno conservador en el poder que considera a la familia uno de los ejes prioritarios de sus políticas. Sin embargo, la irrupción y perduración de una profunda crisis económica da un nuevo significado a sus actuaciones. En la actual fase de reactivación de las políticas neoliberales, el poder político pretende que la familia fortalezca sus funciones de protección y cuidado de sus miembros, en correspondencia con la progresiva disminución de las responsabilidades del Estado. En este contexto, las políticas familiares tienen también una dimensión de género, al poner su foco en la protección de la maternidad y en el papel de las mujeres como madres y cuidadoras. Estas dimensiones, tal como las plantea el gobierno, van más allá de los recortes presupuestarios y tienen un profundo componente ideológico, en tanto la crisis económica constituye una oportunidad para cuestionar e invertir los cambios en los roles de género que se han ido produciendo en los anteriores decenios. Los cambios en la agenda de la familia se corresponden, pues, con cambios en la agenda de igualdad (Lombardo y León, 2014, Paleo y Alonso, 2014).

En el caso de España, las políticas públicas se han asentado firmemente en la idea de que la familia es la institución responsable de sus miembros, lo cual conecta con un imaginario social que asume esta misma concepción prácticamente sin cuestionarla y como una evidencia (Comas d'Argemir, 2014). La noción de familia como ideal imaginario es un concepto muy potente y de gran utilidad, potenciado por las políticas públicas, que alimentan y fomentan la naturalización de la familia, partiendo de la certeza de que esta es la principal suministradora de asistencia y bienestar. Como señala Fineman (2000: 14), "la familia es una construcción ideológica con una particular composición y relaciones de género que permite privatizar la dependencia individual y no considerarla como un problema público".

La familia es una construcción sociocultural pero también ideológica (aunque esto último no siempre sea tan evidente), ya que existe un modelo de familia normativo 
como punto de referencia: el de la pareja heterosexual con hijos/as. Sin embargo las normas familiares han sido muy contestadas en España, los cambios han sido enormes y el concepto de familia se ha ampliado notablemente. La gente se casa menos y lo hace más tarde. Las parejas heterosexuales predominan, pero las del mismo sexo forman también familias. El divorcio ha dado lugar a familias recompuestas, de composición variable de unas semanas a otras según quien tenga la custodia de los hijos. Se puede tener una madre biológica y otra adoptiva. Hay mujeres que crían ellas solas a sus hijos. Hay parejas que viven en distintas poblaciones o en distintos países. Hay personas que viven solas, y seguiríamos... ${ }^{1}$ Ante esta variabilidad, ¿como se delimita lo que es familia y lo que no lo es?

Finalmente son los Estados quienes tienen el poder para definir qué es una familia, a través de su normativa, de sus actuaciones y la definición de las unidades censales. El concepto de familia es variable y depende de cada contexto histórico y social. En España, el concepto de familia se amplió al permitir el matrimonio homosexual y las parejas de hecho pero, para obtener determinadas prestaciones, se han de cumplir ciertos requisitos. De forma bastante generalizada, compartir un mismo domicilio se considera requisito para definir una familia, ${ }^{2}$ aunque en la práctica se generen situaciones muy variables, como es el caso de las familias transnacionales o de las personas que viven solas pero que tienen estrechos vínculos con sus hijos o sus progenitores.

En definitiva, el Estado, con sus mecanismos de poder, no solo define qué es una familia, sino que además actúa de forma jerárquica respecto a ella en sus políticas públicas, decidiendo, por ejemplo, quienes tienen derecho a servicios o prestaciones, o si los hijos de una familia de bajos ingresos o de una madre sola han de pasar a la tutela del Estado o a fórmulas como el acogimiento o la adopción (Marre y San Román, 2012). Los criterios que se aplican en las políticas públicas proceden de un modelo de familia que se ajusta a las condiciones de las clases medias o altas. Son, en definitiva, un acto de clase.

\section{Ideología, desigualdad y género en las políticas familiares en España}

Las propuestas de las distintas formaciones políticas están condicionadas por su ideología, pero también por el contexto social e histórico. Desde el punto de vista ideológico hemos de remarcar que las diferencias entre los partidos conservadores y los de izquierda se traducen a su vez en una horquilla que va de un énfasis en la protección de la familia a un énfasis en la protección de los derechos individuales.

${ }^{1}$ Estos cambios se constatan en los datos elaborados por el Instituto Nacional de Estadística (2001, 2014).

${ }^{2}$ Esta identificación de familia con domicilio no tiene tanto una dimensión jurídica como de actuación político-técnica. Las definiciones censales son muy claras e ilustrativas al respecto y definen la familia, concretamente, como un "grupo de personas que, residiendo en la misma vivienda familiar (por tanto formando parte de un hogar), están vinculadas por lazos de parentesco, ya sean de sangre o políticos, e independientemente de su grado" (Instituto Nacional de Estadística, 2011: 15). 
A su vez, la escasa relevancia de las políticas familiares en España es resultado del rechazo a la herencia del régimen franquista, que sí tuvo una política familiar que puede ser calificada como pronatalista y antifeminista (Valiente, 1996). Tendrán que pasar muchos años para que las políticas familiares dejen de asociarse a los símbolos, medidas y consignas del franquismo. Una revisión de las propuestas y actuaciones de los distintos partidos que operan en el conjunto del Estado español articulando la ideología política con el contexto social y político nos permitirá establecer el marco teórico y contextual en el que situamos nuestro análisis.

En los años de transición democrática y hasta la década de los años noventa hay una práctica inexistencia de políticas familiares en España. Unión de Centro Democrático (UCD), que gobernó entre 1977 y 1982, prestó poca atención a los asuntos de la familia, con escasas o nulas referencias a ella en sus programas electorales y como aspecto relevante cabe mencionar que las prestaciones familiares disminuyeron sustancialmente. Ningún actor social y político pareció interesado en aquellos años en promover un debate que profundizara en objetivos y medidas relacionadas con la política familiar (Valiente, 1996: 157). El régimen franquista estaba demasiado cerca para abordarlo.

Para el Partido Socialista Obrero Español (PSOE) la protección de la familia no fue una prioridad y en cambio puso en primer plano las políticas de igualdad entre mujeres y hombres y las que potenciaban derechos individuales. Cuando llegó al poder a finales de 1982, el PSOE emprendió reformas en el ámbito de la educación, la sanidad y las pensiones, configurando un sistema de acceso universal e individuación de derechos que mejoró las condiciones de vida de las familias (Alberdi, 1997, Valiente, 2013). En las cuatro legislaturas entre 1982 y 1996, se articularon políticas en favor de las mujeres, mientras la familia quedaba desdibujada como objeto de intervención social y política (Salido y Moreno, 2007). Desde el Instituto de la Mujer y sus equivalentes en las Comunidades Autónomas, se potenciaron actuaciones destinadas a que las mujeres tuvieran una mayor presencia en el ámbito laboral y social a través de los Planes de Igualdad de Oportunidades entre Mujeres y Hombres. Si bien los nuevos roles que adquirían las mujeres tuvieron una capacidad transformadora de las familias -en un momento en que la familia tradicional se derrumbaba y muchas personas optaban por formas de convivencia alternativas-, las fuerzas de izquierda no consideraron oportuno promover políticas de apoyo a la familia, identificada con el modelo tradicional que el franquismo tanto había ensalzado y que consideraban que contribuía a la opresión de las mujeres (Subirats, 1998). Esta falta de políticas familiares y de servicios personales supuso, contradictoriamente, un incremento de la presión en trabajo y tensión para las mujeres, que sumaban sus nuevas actividades laborales y sociales a las antiguas responsabilidades familiares que seguían ejerciendo.

El Partido Popular (PP) quiso distinguirse por hacer de la familia un eje importante de su discurso e intervención política ${ }^{3}$. A pesar de que los programas electorales y

\footnotetext{
${ }^{3}$ Nos referimos también a sus antecesores: Alianza Popular, Coalición Democrática y Coalición Popular.
} 
los discursos políticos daban una fuerte importancia a la familia como base de la sociedad, el PP apenas desarrolló actividad parlamentaria en este tema cuando era el principal partido de la oposición. También el PP evitaba ser asociado con el pasado del franquismo, de manera que su inhibición respecto a las políticas familiares contrastaba con la actividad de partidos del mismo signo político en otros países de la UE (Flaquer, 2004b; Valiente, 1996). En la década de los noventa hubo un cambio de actitud, propiciado por la declaración de 1994 del Año Internacional de la Familia por parte de Naciones Unidas. Es significativo en todo caso que el primer acuerdo parlamentario sobre política familiar no lo propiciara el PP, sino Convergència i Unió (CiU), en diciembre de 1997. Es significativo también que por primera vez se alcanzara la unanimidad en este tema, al reconocer las fuerzas políticas de izquierda la necesidad de abordar las políticas familiares en un momento de inflexión, que supuso el redescubrimiento y auge de las políticas familiares en España (Comas d'Argemir, 2012). Se acordó entonces elaborar un Plan Integral de Apoyo a la Familia y, cuatro años después, el primer PIAF fue aprobado, en 2001. ${ }^{4}$

Durante el mandato del PP (entre 1996 y 2004) se impulsaron otras actuaciones vinculadas a la política familiar, además de las mencionadas. Se aprobó una ley de conciliación de la vida familiar y laboral, que introdujo permisos y licencias para el cuidado infantil y los cuidados familiares. ${ }^{5}$ También se introdujeron prestaciones económicas e importantes medidas fiscales, la más conocida de las cuales es la ayuda mensual de 100 euros para las mujeres empleadas con hijos menores de 3 años, aunque las medidas de mayor calado fueron las dos reformas fiscales que incidían en los conceptos que eran objeto de desgravación fiscal y en los tipos impositivos.

Las políticas que impulsó el PP en esos años reforzaban los roles tradicionales de género al entender que solo las mujeres -y no los hombres- habían de conciliar trabajo y familia, favorecían a los grupos solventes al disminuir la progresividad fiscal y mantenían los modelos de familia tradicional, evitando reconocer las nuevas formas familiares emergentes y siendo especialmente beligerantes con las iniciativas que sí lo hicieron posteriormente (como el matrimonio homosexual o las adopciones por parejas homosexuales en Cataluña). Su interés por proteger a las familias numerosas se interpretó por parte de otros grupos políticos como un refuerzo de la asociación tradicional entre familia y procreación, ${ }^{6}$ al tiempo que la modificación de la primera ley de 1988 de reproducción asistida introdujo restricciones consecuentes con las políticas conservadoras que se estaban desarrollando.?

\footnotetext{
${ }^{4}$ A partir de este impulso, el gobierno de la Generalitat de Cataluña (CiU) promovió una ley específica de apoyo a las familias, la Llei 18/2003, de 4 de juliol, de suport a les famílies (ver valoración en Flaquer, 2004a).

${ }^{5}$ Ley 39/1999, de 5 de noviembre, para promover la conciliación de la vida familiar y laboral de las personas trabajadoras.

${ }^{6}$ Ley 40/2003, de 18 de noviembre, de protección a las familias numerosas.

${ }^{7}$ Ley 45/2003, de 21 de noviembre, sobre técnicas de reproducción asistida. El objetivo de la modificación de la ley era limitar la acumulación de preembriones sobrantes de los procesos
} 
En las siguientes dos legislaturas (2004-2011), última etapa de gobierno del PSOE, este impulsó políticas de género: se reguló el matrimonio entre personas del mismo sexo $^{8}$, se aprobó la Ley de Igualdad (que introdujo un permiso de paternidad) 9 , la Ley de Dependencia ${ }^{10}$, la Ley de adopción internacional ${ }^{11}$ y la Ley contra la violencia de género $^{12}$. La novedad, en el caso del PSOE, es que asumió la necesidad de intervenir en políticas familiares, hasta el punto de que Mercedes Cabrera, ministra de Educación, Política Social y Deporte, declaró poco después de su nombramiento en el año 2008 que la familia sería el eje central de las políticas sociales, dando así un giro copernicano respecto a la actitud que había mantenido su partido hasta entonces. A pesar del impulso que ello implicó en las políticas sociales que inciden en la familia, no se llegaron a alcanzar los niveles de prestaciones y de servicios existentes en otros países europeos. ${ }^{13}$ La decisión -muy polémica- de incluir una ayuda de $2.500 €$ por nacimiento de hijo/a (el llamado "cheque-bebé") instaurada en 2007 fue eliminada en enero de 2011 por los recortes presupuestarios.

Un breve repaso de los programas de los partidos de ámbito español que concurrieron a las elecciones generales de 2015 permite resituar el peso que se otorga a la familia en el espectro político. Pasado ya medio siglo desde la muerte del dictador, las políticas familiares se plantean de forma abierta, aunque no con la misma intensidad y con notables diferencias según el espectro ideológico. El PP dedica un apartado específico de su programa a la protección social, jurídica y económica de la familia, y además de las propuestas contenidas en el PIAF plantea que la perspectiva familiar impregne todas las políticas. Ciudadanos propone numerosas medidas de apoyo a las familias, apuesta por la regulación de la gestación subrogada y pone en cuestión la normativa

de fertilización. Dada la baja tasa de éxito de los tratamientos de fecundación, el límite de tres preembriones obligaba a las mujeres a "sufrir de nuevo el doloroso proceso de estimulación ovárica si el tratamiento no da resultado con esos tres intentos" (Real Decreto 1720/2004). Con la modificación de la ley se deja al criterio clínico de los profesionales determinar el número de ovocitos a fecundar en cada caso.

${ }^{8}$ Ley 13/2005, de 1 de julio, por la que se modifica el Código civil en materia de contraer matrimonio.

${ }^{9}$ Ley Orgánica 3/2007, de 22 de marzo, para la igualdad efectiva de mujeres y hombres.

${ }^{10}$ Ley 39/2006, de 14 de diciembre, de Promoción de la Autonomía Personal y Atención a las personas en situación de dependencia.

${ }^{11}$ Ley 54/2007, de 28 de diciembre, sobre Adopción Internacional.

${ }^{12}$ Ley Orgánica 1/2004, de 28 de diciembre, de Medidas de Protección Integral contra la Violencia de Género.

${ }^{13}$ En el año 2007 el gasto en protección social en España fue de un 20,5\% del PIB, por debajo de la media de los países de la UE15 $(27,7 \%)$ y a bastante distancia del de Francia, que alcanzó un 30,5\%. Por lo que respecta al gasto en familia e infancia, España dedicó un 1,2\% del PIB, el mismo porcentaje que Italia y Portugal, mientras que la media de la UE15 fue de 2,1\% y Dinamarca alcanzaba el 3,7\% (elaboración propia a partir de los datos de Eurostat). Para una comparativa sistemática de las políticas familiares en contexto europeo en años precedentes a los que tratamos, consultar Alberdi (1997) y Flaquer (2004b). 
sobre violencia de género, siendo estas dos últimas cuestiones fuertemente contestadas desde el feminismo. El PSOE mantiene su prioridad en las políticas de igualdad, y en un apartado sobre tendencias demográficas es donde plantea desplegar "políticas amistosas para las familias", que niega identificar con políticas natalistas, sino con el desarrollo de preferencias reproductivas de la ciudadanía. En el programa de Podemos no hay ningún apartado sobre familia, aunque sí algunas medidas concretas (en cambio, en el programa marco de las elecciones autonómicas, sí aparece un breve apartado). Tampoco hay referencia explícita a las políticas familiares en el programa de IU, aunque sí se proponen algunas medidas en el marco de propuestas feministas.

Los cambios en el contexto social y político permiten comprender los motivos por los que durante años la familia no fue objeto de intervención pública en España y la progresiva asunción por parte de todo el espectro ideológico de la necesidad de promover políticas familiares. Hemos podido comprobar que en este proceso se mantiene la horquilla entre las opciones que priorizan la protección de la familia como institución y las que priorizan la protección de los derechos individuales. Trataremos de mostrar en nuestro análisis como estos componentes ideológicos se expresan en el PIAF e inciden en la agenda de la igualdad de género y de la igualdad social.

\section{Metodología}

Los documentos políticos ofrecen un interesante campo de investigación como expresión de un tipo de poder que queda oculto detrás de un lenguaje técnico que difícilmente se somete a escrutinio. Constituyen un género propio, basado en intenciones y compromisos, que está orientado a complacer y persuadir más que a informar o describir y en esto consiste su poder (Apthorpe, 1997: 45). El lenguaje político incorpora determinadas asunciones que aparecen como evidentes e incuestionables y que actúan como máscaras que ocultan determinadas relaciones y formas de dominación (Gledhill, 1999). No es, pues, un lenguaje neutro, ya que está relacionado con los mecanismos de poder, crea categorías, les asigna significados, jerarquiza y construye al individuo como sujeto (Shore y Whright, 1997: 18).

Para abordar el análisis del PIAF 2015-2017 hemos tenido en cuenta el documento oficial aprobado por el Consejo de Ministros y también los dos borradores que lo han precedido. Este planteamiento ha permitido tener una perspectiva más completa del proceso de elaboración, ya que los diferentes textos se superponen de forma estratificada, lo que permite analizar los cambios realizados, los nuevos énfasis o incluso también las dimensiones más conflictivas. Asimismo lo comparamos con el Plan Integral de Apoyo a la Familia 2001-2004 (en adelante PIAF 2001-2004).

Adoptamos como metodología el análisis crítico del discurso, que entronca con los paradigmas interpretativistas de la antropología social, así como con las consideraciones del discurso como práctica social (Gasper y Apthorpe, 1996; van Dijk, 2000). Asimismo, interpretamos los contenidos discursivos en el marco de los componentes culturales presentes en el contexto social y político en que se genera el PIAF 2015-2017. Hemos seguido en este sentido la siguiente estrategia metodológica: 
1. Identificar los enfoques conceptuales (frames). Este procedimiento se relaciona con el problema de investigación que hemos definido, en que vinculamos las políticas familiares a las nociones de biopolítica, género y desigualdad. También se vinculan con la ideología, inherente al propio hecho de que se trata de un documento político.

2. Identificar las categorías discursivas o ideas clave que constituyen el eje principal del discurso. Nos centramos en las que se encuentran en la superficie de la estructura textual, pero también en las que se hallan subyacentes y no explícitas.

\section{El Plan Integral de Apoyo a las Familias 2015-2017 en el contexto de las políticas familiares}

El 14 de abril de 2014, el presidente del gobierno español, Mariano Rajoy, y la ministra de Sanidad, Servicios Sociales e Igualdad, Ana Mato, anunciaban que el gobierno estaba trabajando en un Plan Integral de Apoyo a la Familia, que sería presentado tras el verano en su versión definitiva. Un primer borrador inicial muy esquemático de 17 páginas fue distribuido a la prensa y las asociaciones familiares, entre otras entidades, como parte del proceso de elaboración. Que la máxima autoridad política española -poco dada a la comparecencia pública- estuviera presente en la rueda de prensa junto a la ministra fue un indicador de la gran importancia otorgada al Plan, como recogieron los medios de comunicación que difundieron la noticia.

El Plan estaba siendo elaborado por la Comisión Técnica Interministerial de Familia, ${ }^{14}$ que trabajaba en realidad un segundo PIAF, pues ya hubo un primero, vigente entre 2001 y 2004. El 7 de noviembre de 2014 se presentó un segundo borrador de 79 páginas, prácticamente el texto definitivo, titulado Plan Integral de Apoyo a la Familia 2014-2017, borrador de trabajo. En este texto, que también fue distribuido entre las asociaciones familiares aunque no tuvo la proyección mediática inicial, se detallaban los objetivos, medidas y líneas estratégicas. Finalmente, el 14 de mayo de 2015 se aprobó el texto definitivo del PIAF 2015-2017 por parte del Consejo de Ministros. ${ }^{15}$ El documento final tiene 86 páginas, y en él se actualizan datos, se incorporan los resultados de la Encuesta sobre Opiniones y Actitudes sobre la Familia realizada por el Centro de Investigaciones Sociales (CIS) y se modifican algunos de los contenidos y propuestas iniciales.

El valor político del documento que analizamos estriba en que, en él, el gobierno conservador del Partido Popular (PP) se reivindica como el abanderado de las políticas familiares en España. Se subraya que el primer PIAF 2001-2004 (elaborado también durante un gobierno del PP) fue el primer y único plan integral de apoyo a la familia y que, desde que el Partido Socialista Obrero Español (PSOE) ganó las elecciones

${ }^{14}$ La Comisión Técnica Interministerial para elaborar el PIAF, creada por el Consejo de Ministros, se constituyó el 16 de abril de 2013 bajo la presidencia del Secretario de Estado de Servicios Sociales e Igualdad.

${ }^{15} \mathrm{El}$ documento definitivo llegó con retraso respecto a lo que estaba previsto, por lo que tuvo que modificarse el período de implantación, que pasó a ser de 2015 a 2017 (y no desde 2014). 
en 2004, las familias no fueron prioritarias, ya que durante los ocho años de gobierno socialista no se redactó un nuevo PIAF: "Es durante la presente legislatura cuando la familia vuelve a estar presente en la agenda política del Gobierno" (PIAF 2015-2017, p. 5).

Tal como hemos podido comprobar en el apartado anterior, la alternancia de gobierno entre el PSOE y el PP desde el año 1982 ha comportado la alternancia de distintas formas de intervención política respecto a la familia (Comas d'Argemir, 2012; Salido y Moreno, 2007). El PP ha hecho de la familia, percibida desde un enfoque tradicional como una unidad social primaria, un eje esencial de sus actuaciones en política social. Las nuevas formas familiares no consiguen romper o tan siquiera matizar este esquema, pues se incluyen en el universo familiar como "otras" formas de familia, lo que refuerza la centralidad de la familia tradicional. El PSOE y los partidos de izquierdas (como IU, por ejemplo) consideran que el apoyo a la familia se hace desde las políticas de bienestar y, en todo caso, apoyan las responsabilidades familiares y no la institución familiar. No obstante, dado que en los últimos años estos partidos decidieron abordar las políticas familiares, el PP necesita reivindicarse como el verdadero garante protector de la familia.

Es en el contexto de la pugna con el PSOE por un sector estratégico (demográfico y electoral) donde debemos situar el interés del PP en remarcar su permanente e incondicional interés por apoyar a las familias, tal como hace en el PIAF. Es un "compromiso político del gobierno", se dice, al tiempo que no se hace referencia alguna a las iniciativas del PSOE en ese terreno sino que se minimizan -o ignoran- al señalar que "la familia no había sido objeto de una acción integrada y transversal". Por ello, se reivindica que la familia vuelve a estar presente como prioridad y se insiste en que las actuaciones del gobierno "tienen como eje central a la familia (incluyendo de forma especial el apoyo a la maternidad)" (PIAF 2015-2017, p. 5).

La relevancia de esta clase de actuaciones, inscritas en recomendaciones de alcance internacional, se muestra en el mero hecho de efectuar un informe para la Comisión Europea (Arriba y Moreno, 2015). Como comentario del Plan, el informe señala tres grandes debilidades: la ausencia de debate público sobre las medidas propuestas, los problemas de género por la perpetuación del rol de las mujeres como cuidadoras y la falta de recursos económicos para llevar adelante el Plan. Añadiremos nosotras que muchas de las actuaciones previstas conciernen en realidad a Comunidades Autónomas y a gobiernos locales, sin que se haga alusión a su posible aceptación y compromiso.

\section{Objetivos y líneas estratégicas del PIAF 2015-2017}

El objetivo del PIAF 2015-2017 es desarrollar una política específica y explícita para la familia a partir del reconocimiento de su valor primordial en la sociedad y la necesidad de que los poderes públicos la apoyen para que pueda asumir plenamente sus responsabilidades. Los objetivos del Plan se fundamentan en distintos documentos, recomendaciones e informes elaborados por Naciones Unidas, el Consejo de la Unión Europea, el Comité Económico y Social Europeo y otras instituciones supranacionales y nacionales e intentan atender a los retos sociales a que se enfrentan Europa y España 
en un período de "ansiedad demográfica" (Carrasco, Borderías y Torns, 2011; Comas d'Argemir, 2015; Fonseca, Marre y San Roman, 2015; Kohler, Billari y Ortega, 2002; Marchesi, 2012; Marre, 2011; Mishtal, 2014).

El análisis de un texto técnico-normativo como el PIAF que orienta la actuación política es importante no solo por las medidas que introduce, sino también por las premisas y supuestos que lo inspiran. A veces son tan implícitos que ni se mencionan; pero hay también silencios. En tanto el PIAF 2015-2017 explicita con bastante detalle los objetivos generales y principios que guían el Plan, los silencios resultan mucho más estridentes. El texto empieza con un diagnóstico de la situación socioeconómica de las familias en España. A partir de datos demográficos, se explican los cambios en la composición de la población y en la formación y características de las familias, con especial atención al descenso de la natalidad y sus causas. Se explican también las características de los hogares (tamaño y composición) y el peso relativo de las familias monoparentales y con personas con discapacidad. Finalmente, se dedica un apartado a indicadores de calidad de vida (renta y consumo de los hogares, vivienda, nivel educativo, violencia de género). Resulta altamente llamativo en este diagnóstico que haya aspectos cruciales en la vida familiar que no se mencionan o se hace genéricamente. Nos referimos, básicamente, a cinco hechos sociales de enorme importancia: el desempleo, la pobreza infantil, el incremento de las desigualdades entre familias, los desahucios y la diversidad familiar.

Respecto al primero de ellos, se menciona únicamente la "fuerte pérdida de empleos que se inicia en la legislatura anterior, y que desde el inicio de 2014 ha invertido su tendencia “ (PIAF 2015-2017, p. 19), lo que sirve para explicar el descenso del nivel de renta en pocos años (de $30.045 € /$ año en 2008 a $26.775 € /$ año en 2013) y el descenso del gasto medio por hogar (cifrado en 4.613 euros menos en 2013 que en 2008). En el PIAF 2015-2017, no se comenta como el desempleo afecta a las familias: no hay ninguna referencia al número de parados ni a la magnitud del paro de larga duración (3,5 millones de desempleados con más de un año sin trabajar). Tampoco parece que sea relevante, ya que no se cita, la existencia a finales del 2013 de 1.832 .300 hogares en que todos sus miembros están sin empleo, ni de los casi 700.000 hogares en que no hay ningún ingreso. ${ }^{16}$ Son magnitudes demasiado elevadas como para ser silenciadas o ignoradas, teniendo en cuenta el grave impacto negativo que suponen para las familias, tal como se demuestra en el ler Informe sobre la Desigualdad en España 2013 (RuizHuerta, 2013).

Igualmente destacable es el silencio en torno a la pobreza infantil, que no se menciona en el diagnóstico, aunque aparece después en los objetivos de forma tangencial. Los datos del informe de la UNICEF (2013) sobre bienestar infantil en 29 países del mundo, son lacerantes. En 2008, España se situaba en el quinto lugar del mundo en bienestar infantil y, cinco años después, en 2013, en el puesto 19. Una tendencia ratificada por el informe de 2014, según el cual cada vez hay más niños/as pobres y cada vez lo son más. El PIAF 2015-2017 no habla de pobreza infantil en su diagnóstico ni de privación,

${ }^{16}$ Datos del Instituto Nacional de Estadística. 
a pesar de que un $20 \%$ de niños y niñas sufren privaciones materiales y vulnerabilidad económica. El escaso desarrollo de las políticas públicas es una de las causas por las que España esté a la cabeza de Europa en pobreza infantil, especialmente en un momento de fuertes tasas de desempleo. El gasto social en protección familiar en España sigue siendo de los más bajos de la Unión Europea con el 1,5\% del PIB, frente a porcentajes superiores al 3\% en muchos países de Europa. Como señala Rodríguez Cabrero (2014), la filosofía de la inversión social no es un factor constitutivo de las políticas públicas y está subordinada a la presión de la emergencia social.

Por otra parte, el PIAF 2015-2017 no considera el incremento de las desigualdades entre familias sino solo la desigualdad debida a las situaciones internas de las familias, como tener personas con discapacidad, por ejemplo. No se trata solo de tener cubiertas las cuestiones materiales, sino de la brecha de desigualdad que cada vez se profundiza más, un terreno en que España ocupa el último lugar de los países más ricos (RodríguezHuerta, 2013). Ningún niño o niña debería ver hipotecado su futuro por las dificultades económicas, de salud o relacionales de sus familias, pero estas no constituyen una prioridad.

El texto también obvia el importante número de familias que han sido desahuciadas de su hogar. El apartado de diagnóstico del PIAF 2015-2017 hace referencia a la vivienda como indicador de calidad de vida y, sorprendentemente e irónicamente, solo subraya el incremento del porcentaje de familias que tienen vivienda en propiedad. Ni una sola mención al grave problema de familias desahuciadas en los años en que justamente se redacta el PIAF: un total de 101.034 lanzamientos en el 2012, de los que 30.050 corresponden a la vivienda habitual, según datos del Consejo General del Poder Judicial (2013)

Un quinto silencio llamativo es el que hace referencia a la diversidad familiar existente que, si bien se menciona en los objetivos y el término "diversidad familiar" se añade varias veces en el texto definitivo, se hace de forma contrastada al modelo normativo de familia. Es un silencio coherente con la idea de familia del gobierno conservador, que es una familia tradicional, heterosexual y orientada a la procreación. Tal como tendremos ocasión de analizar, hay una contradicción presente en el texto entre denominar la diversidad familiar y asumirla como tal. Como no se puede negar la existencia de una gran heterogeneidad familiar, se la trata como algo diferente de la familia normalizada, es decir, que el diagnóstico no considera relevante constatar que aquello que caracteriza a la familia actual es justamente su diversidad.

Los objetivos y principios del borrador del PIAF 2015-2017 se despliegan en diez puntos, a los que se añaden siete líneas estratégicas que incluyen 228 medidas a promover. Agruparemos todo ello en cinco grandes apartados que expresan los enfoques conceptuales del documento, para comentar las ideas clave que aparecen en el mismo.

\subsection{La familia como unidad social primaria}

El PIAF 2015-2017 considera a la familia como la unidad social primaria de la sociedad y modelo de referencia con el que compara otras realidades familiares y en distintas partes del documento se legitima esta idea aludiendo a la elevada valoración que los españoles hacen de la familia. Este concepto de familia aparece de forma transversal en 
todo el documento y la protección de la familia como institución constituye uno de los objetivos esenciales del PIAF. La idea de solidaridad es clave en el papel central que se otorga a la familia para hacer frente a las situaciones de necesidad.

El texto inicia su redactado mencionando a la familia

como unidad básica de articulación social, escuela de valores, lugar privilegiado para la transmisión del patrimonio cultural, agente de cohesión e integración social, factor clave de la solidaridad intergeneracional, y espacio vital para el desarrollo y bienestar de sus miembros, a los que los poderes públicos deben apoyar para que puedan asumir plenamente sus responsabilidades (PIAF 2015-2017, p. 4).

Y dice poco más adelante:

La familia desempeña un papel económico y social e incluso político, imprescindible para la cohesión y sostenibilidad social. Es a la vez necesaria para lograr estabilidad y crecimiento económico sostenido; lleva a cabo una función educativa clave para la configuración de una sociedad equilibrada; y desempeña una función de solidaridad entre personas y generaciones que le convierten -junto con el sector público- en una institución capaz de redistribuir rentas y de hacerlo de manera totalmente altruista para la sociedad (PIAF 2015-2017, p.4)

Esta descripción coincide plenamente con la que aparecía en el PIAF 2001-2004. Se describen hechos constatables en la realidad, como es la solidaridad familiar o las funciones de la familia, pero la lectura integral del documento deja ver que lo que se propone es proteger este papel de la familia para reforzar la responsabilidad de sus miembros en el marco de un progresivo retroceso de la implicación del Estado. Es la solidaridad entre adultos la que ha de resolver las necesidades vinculadas a la procreación, la crianza o el cuidado de adultos dependientes. Lo que es una constatación se convierte así en prescripción: son los principios tradicionales del parentesco los que han de resolver las situaciones de necesidad, mientras que el Estado se limita a "apoyar". Las premisas que enfatizan el papel de la familia como factor de cohesión social y de solidaridad intergeneracional se sustentan pues en una imagen tradicionalista y familiarista de las políticas públicas, y expresan la falta de voluntad real de desarrollar políticas sociales avanzadas.

La imagen tradicionalista de la familia se proyecta también cuando el PIAF 20152017 se refiere a las familias metafóricamente como "cimientos del edificio social" (p. 27), o como "el principal amortiguador de los efectos que se derivan de dificultad económica y social" (pp. 8-9 y 27). La familia es, efectivamente, una malla de protección social, muy importante en los países de régimen mediterráneo de bienestar. Nuevamente podemos observar que el lenguaje político, presentando situaciones que corresponden a la realidad, prescribe también como debería ser esa realidad.

La elevada valoración de la familia tradicional se incrementa cuando el PIAF 20152017 presenta a las familias numerosas como ejemplo de familia por excelencia: "Se debe reconocer la contribución y aportación de las familias, especialmente con mayor número de hijos, como elemento de cohesión social y de desarrollo de la solidaridad 
intergeneracional" (p. 28). El argumento es explícito: las familias numerosas no solo cumplen sus funciones de procreación, sino que también amplían la red de solidaridad y apoyo. Obvia que ello solo es posible cuando lo material está mínimamente resuelto, lo que no siempre es el caso.

\subsection{La familia y las "otras" familias}

El PIAF 2015-2017 no define qué es una familia y reconoce diversos modelos de familia. En este sentido es integrador, ya que incorpora las familias de personas homosexuales, transexuales y bisexuales (LGTB), reconstituidas, inmigrantes, familias adoptivas y de acogida, pero en cambio estas familias no se consideran parte de las familias normalizadas. Las ideas claves en este marco conceptual no se encuentran tanto en elementos discursivos como en la clasificación binaria entre familias: las familias, a secas, que no hay describir ni añadir marcadores en su denominación porque se da por supuesto en qué consisten, y las familias que sí requieren denominaciones específicas y que a efectos analíticos agrupamos como "otras familias". Resulta significativa, en este sentido, la clasificación binaria que hace el propio documento entre distintos tipos de familias cuando despliega el objetivo 7 de "ofrecer apoyo preferente a familias que se encuentran en situaciones especiales".

Así, el apartado dedicado a "Medidas de apoyo a familias numerosas, familias monoparentales y familias con personas con discapacidad" (p. 68) se refiere a familias que responden al modelo normativo, pero con la especificidad de una sobrecarga en sus responsabilidades que les dificulta su ejercicio, sea por el elevado número de hijos, la monoparentalidad o la atención a personas dependientes o con alguna discapacidad. Las medidas van orientadas a apoyar a estas familias mediante descuentos, medidas fiscales o apoyando el cuidado familiar y, en este sentido, recoge objetivos y propuestas similares a las del primer PIAF 2001-2004.

Un segundo grupo de medidas está dirigido a "otros colectivos familiares con especiales necesidades" (p. 72), cuyo elemento común es que se alejan de la normalidad, mezclando situaciones realmente muy distintas como familias de lesbianas, gais, transexuales y bisexuales (LGTB), familias afectadas por la violencia, inmigrantes, empleados públicos en el exterior, madres reclusas o familias ambulantes que viven del circo. Son, pues, un tipo de familias que se distingue de las anteriores por alejarse de lo que se considera normal, en su calidad de familias minoritarias, con problemas, diferentes. ${ }^{17}$ El PIAF 2015-2017 no reconoce la diversidad familiar como algo normalizado, sino como excepcional. Y es que el PIAF no trata de la misma forma los distintos modelos familiares con situaciones especiales, sino que los jerarquiza. Desde esta concepción, es difícil normalizar las "familias diferentes", a pesar de que la realidad muestra que hay cada vez más niños y niñas que viven en familias "no tradicionales", lo que no debería contribuir a su estigmatización (Comas d'Argemir, 2013). Las políticas

${ }^{17}$ Una muestra de que las familias de personas homosexuales, transexuales y bisexuales (LGTBI) no se igualan con el resto es que desde el año 2013 se ha excluido de la cartera de servicios las técnicas de reproducción asistida para las mujeres lesbianas y mujeres solas que quieran ser madres, sin que el PIAF lo modifique. 
públicas deberían reconocer e integrar la diversidad como un valor en sí mismo, cosa que no hacen los contenidos ideológicos que guían el PIAF 2015-2017.

\section{Proteger la maternidad}

La protección de la maternidad es una de las dimensiones con mayor contenido ideológico en las políticas del PP. Mientras los partidos de izquierda han puesto el énfasis en la salud sexual y reproductiva de las mujeres, generalmente asimilándola a la anticoncepción y considerando a la maternidad fruto de una elección responsable, el partido conservador gobernante se centra exclusivamente en el apoyo a la maternidad, sin referencia alguna a la posibilidad de programarla, retrasarla o impedirla, de acuerdo con las necesidades y proyectos vitales de las mujeres y sus parejas, si las tienen. Dos ideas clave aparecen en el documento en relación a este tema. Una, bien explícita y detallada, es la protección de la maternidad, que cuenta con una amplia profusión de medidas. Otra, silenciada, es el aborto voluntario, que es contrarrestado mediante medidas disuasorias que se dirigen especialmente a mujeres adolescentes y a mujeres en situación de vulnerabilidad, con la finalidad de proteger al no nacido. Ambas son expresiones del ejercicio del biopoder, que controla el cuerpo de las mujeres y modela las subjetividades potenciando la figura de la mujer-madre.

El PIAF 2015-2017 atribuye a la maternidad gran importancia, tanto por lo que respecta a su incidencia en la vida de las mujeres, como por sus efectos sociales y demográficos. Estas dimensiones pueden ser compartidas desde distintas ideologías, pero la especificidad del PP se halla en la especial relevancia que otorga a la maternidad como eje central de la vida familiar. Así, las actuaciones que se prevén en el PIAF 2015-2017 no van dirigidas a las mujeres, sino a las mujeres-embarazadas o a las mujeres-madres. El único objetivo que trata de "impulsar la educación afectivo sexual y la prevención de embarazos no deseados" (p. 50) no se traduce en medidas concretas, sino genéricas.

Este apartado del PIAF es el que ha tenido más cambios en su planteamiento, de manera que los dos borradores y el texto definitivo del PIAF 2015-2017 presentan diferencias significativas desde el punto de vista ideológico. El tema conflictivo es el del aborto. Así, el borrador inicial incluía un punto que fue eliminado posteriormente. Se trata del objetivo consistente en la "protección del no nacido durante su crecimiento y desarrollo, estableciendo y proporcionando a la madre la información e instrumentos necesarios que le faciliten continuar con su embarazo" (PIAF primer borrador, p.16). Este enunciado tenía un contenido muy parecido al del Proyecto de ley de protección de la vida del concebido y de los derechos de la mujer embarazada que quería modificar la ley vigente sobre el aborto ${ }^{18}$. Este proyecto de ley, finalmente retirado después de dos años de debate y contestación social, culminó con la dimisión del Ministro de Justicia que lo había impulsado, y se retiró del segundo borrador del PIAF el objetivo

${ }^{18}$ Ley Orgánica $2 / 2010$, de 3 de marzo, de salud sexual y reproductiva e interrupción voluntaria del embarazo. 
que pretendía proteger al no nacido. Sin embargo, los principios ideológicos que inspiraban ese objetivo se mantienen en las medidas propuestas. El término 'aborto' o el de 'interrupción voluntaria del embarazo' no aparecen nunca, y en texto definitivo se elimina incluso una expresión del segundo borrador (p. 47) que podía evocar el aborto, como es que se identificarían factores de riesgo, complicaciones del embarazo o anomalías fetales para "minimizar sus consecuencias", lo que se sustituye por "superar las dificultades que pudieran impedir un claro apoyo a la maternidad" (PIAF 20152017, p. 52 medida 73). También se elimina del texto definitivo que la adopción y el acogimiento sean "alternativas eficaces para permitir a las mujeres gestantes seguir con su embarazo" (PIAF segundo borrador, p. 44), aunque sí contempla, indirectamente, facilitar la adopción. Así, se incluye, por ejemplo, "apoyar y fomentar el desarrollo de iniciativas sociales que tengan como finalidad el apoyo a mujeres embarazadas, menores en gestación y a las madres en situación de postparto o lactancia que presenten situaciones de vulnerabilidad o riesgo social" (p. 51, medida 68) y, como fruto de ello, "coordinar (con las CCAA) las actuaciones en casos de entrega de un recién nacido en adopción cuando su madre y entorno más próximo no puedan hacerse cargo del mismo" (p. 54, medida 86).

La preocupación por el envejecimiento de la población y por la baja natalidad aparece de forma reiterada en el PIAF 2015-2017, pero el texto definitivo presenta un cambio significativo respecto a los borradores previos y es que se menciona de forma explícita el objetivo de "incentivar la natalidad" (pp. 48 y 49). En este sentido se añade una medida (la 80), para instaurar un complemento en las pensiones de mujeres que hayan tenido dos o más hijos. Aparte de los problemas económicos y políticos para implementar esta medida (supone modificar los Pactos de Toledo), resulta de dudosa efectividad para animar a las mujeres a tener más hijos.

El apartado de diagnóstico del PIAF 2015-2017 da numerosos datos sobre el escaso número de nacimientos en España, su descenso continuado en las tres últimas décadas, el retraso en la edad de la maternidad y las bajas tasas de fecundidad que en 2013 no llegaban a 1,3 hijos por mujer (p. 15). Se trata de una "infertilidad estructural" (Marre 2009) que tiene relación con la dificultad para abordar y desarrollar proyectos de maternidad a causa de la precariedad laboral, los elevados precios de la vivienda y la ausencia de políticas y servicios públicos para atender la pequeña infancia. Sorprendentemente el PIAF 2015-2017 no analiza estas causas estructurales que provocan el retraso de los proyectos de maternidad e incluso impide tener los hijos deseados (Alvarez, 2013; Briggs, 2012; Comas d'Argemir, 2012; Marre, 2011). Las medidas propuestas se concentran en promover un "entorno favorable a la vida familiar", a través de incrementar el conocimiento del papel de las familias, mejorar su protección jurídica, lograr ciudades sostenibles y competitivas, mejorar los tejidos residenciales y recuperar los conjuntos históricos, mejorar la confianza y seguridad en internet, promover actividades de ocio, culturales y deportivas en familia, facilitar la emancipación de los jóvenes y fomentar la solidaridad intergeneracional (PIAF 20152017, pp. 50-51). Si bien es evidente que los objetivos mencionados inciden en la calidad de vida familiar, no se abordan los problemas que provocan la "infertilidad estructural" en España. Y es en este terreno donde se proyecta una vez más la ideología 
conservadora del PIAF: la familia es una institución a proteger por ella misma desde bases ideológicas y valores morales, un contexto de la familia donde cobra sentido la protección de la maternidad, columna vertebral de la institución familiar.

\section{Conciliar y educar. Corresponsabilidad y parentalidad positiva}

La corresponsabilidad y la parentalidad positiva constituyen ideas clave en el PIAF 2015-2017, siendo importantes dimensiones de la calidad de vida familiar que implican esfuerzo y compromiso por parte de los miembros de la familia en el ejercicio de sus funciones. Son dimensiones hoy ampliamente reconocidas como necesarias y recomendadas por los organismos internacionales.

Fomentar el empleo de madres y padres requiere posibilitar la conciliación laboral y familiar, lo cual abarca los permisos maternos y paternos para atender a hijos e hijas, facilidades de reincorporación a la vida laboral, atención a la infancia y disponibilidad de tiempo para cuidar personas adultas dependientes. Hablar de conciliación significa reconocer un conflicto que se expresa en la vida cotidiana y afecta de forma desigual a hombres y a mujeres (Bustelo, 2006; Carrasquer, Massó i Martín, 2007; Pazos, 2013; Recio, 2015; Torns, 2005). Se trata, pues, de establecer cuál es el compromiso público en la atención a la infancia y personas dependientes, y como repartir el cuidado entre hombres y mujeres en corresponsabilidad mutua.

Sin embargo, en España, la conciliación se ha concebido como un problema de mujeres. La Ley de conciliación de 1999 resulta insuficiente -a pesar de que algunos aspectos se revisaron posteriormente-ya que no confronta el hecho de que las mujeres son quienes padecen discriminación laboral y ven afectados sus proyectos de maternidad ${ }^{19}$. Se ha comprobado que los países europeos con mejores condiciones de empleo para las mujeres tienen las tasas de fecundidad más elevadas (tal es el caso de Francia, Islandia, Suecia y Noruega), mientras que ocurre lo contrario en los países con mayor precariedad e inestabilidad laboral, como Grecia, Italia, España y Polonia (Adsera, 2011; Bernardi, 2005; Krause y De Zordo, 2012; Marre, 2011; Mishtal, 2014; Morgan y Taylor, 2006). Las jornadas a tiempo parcial han sido más una estrategia utilizada por las empresas para su organización interna que un apoyo efectivo a la conciliación y a la parentalidad positiva. Por otro lado, son políticas pensadas para quien cuenta con una situación laboral estable y relativamente satisfactoria (contrato indefinido, retribución suficiente), al tiempo que no contemplan realidades de convivencia familiar como las parejas de hecho, familias monomarentales u homosexuales. La desigual distribución del trabajo entre hombres y mujeres, lejos de reducirse, se ha acentuado, pues la incorporación de las madres a la obtención de ingresos es muy superior a la de la implicación de los padres en los cuidados domésticos. Por ello, la conciliación acaba configurando un desequilibrio que recae principalmente en las mujeres, en permanente tensión entre sus responsabilidades de cuidado familiar y sus carreras y oportunidades profesionales.

${ }^{19}$ Ley 39/1999, de 5 de noviembre, para promover la conciliación de la vida familiar y laboral de las persones trabajadoras. 
La introducción del permiso de paternidad de trece días supuso una medida interesante y resaltable creada por la Ley de Igualdad con el objetivo de alcanzar las cuatro semanas de permiso en 2013. Un equipo de investigación de la Universidad Complutense de Madrid realizó, a petición del Instituto de la Mujer, una evaluación de este permiso para establecer si ha fomentado una mayor corresponsabilidad masculina en el cuidado de hijos e hijas. Si bien la conclusión fue que existe una asociación positiva entre el uso del permiso de paternidad y una mayor implicación de los padres en los cuidados infantiles (Escot y Fernández, 2012: 123), la brecha mujer-hombre en el uso de los permisos parentales sigue siendo muy grande. La tasa de permisos paternos sobre el total de hombres y mujeres en permiso es del $11,34 \%$, baja aunque superior a la del periodo anterior a la reforma en que los hombres utilizaban una parte del permiso de la madre (5,99\%). Los autores del informe señalan que esta brecha está causada por la corta duración del permiso de paternidad y recomiendan avanzar hacia la plena igualdad de género en el acceso a los permisos parentales y estimular a los varones a implicarse más en los cuidados infantiles y el trabajo familiar. A iniciativa de La Plataforma por Permisos Iguales e Intransferibles de Nacimiento y Adopción (PPIINA), la Comisión de Igualdad del Congreso de Diputados aprobó por unanimidad, en octubre de 2012, una proposición no de ley que insta al gobierno a "avanzar hacia la equiparación de permisos de nacimiento, adopción y acogimiento entre ambos progenitores, de forma que ambos cuenten con el mismo periodo personal e intransferible, eliminando las disfunciones existentes en la legislación actual al respecto y, singularmente, evitando la subrogación de derechos que han de ser individuales". Aunque aprobada, esta proposición está en vía muerta. Y el PIAF ni la menciona. El PP, además, es el único partido que no la incluye en sus programas electorales.

Las nuevas políticas deberían estimular una conciliación corresponsable y realista a tres niveles: corresponsabilidad entre hombres y mujeres para equilibrar el esfuerzo pero también las satisfacciones relacionadas con el cuidado y la parentalidad positiva; corresponsabilidad entre las personas y las empresas para introducir prácticas organizativas que favorezcan los intereses de cada parte; y corresponsabilidad con la Administración responsable de subministrar los servicios y recursos que permitan la conciliación.

El PIAF 2015-2017 aborda la corresponsabilidad entre hombres y mujeres en elámbito familiar como "una exigencia derivada del aún desigual reparto en la asunción de tareas domésticas o de cuidado" (p. 42). Sin embargo, las medidas propuestas son genéricas y poco concretas. En este sentido, no incluye ninguna novedad respecto a las actuaciones de las empresas y, notablemente, no hay ninguna medida que estimule más activamente a los hombres a la conciliación y a reforzar su compromiso en la vida familiar, excepto alguna referencia al abordar la parentalidad positiva. En el PIAF-segundo borrador no se mencionan las desigualdades entre hombres y mujeres en materia de empleo o en el trabajo familiar no remunerado, como si ambos partieran de unas mismas condiciones, cosa que se corrige en el texto definitivo. Pero este cambio no modifica las propuestas, que son meramente declarativas. Podemos considerarlo como otra forma de expresión de la biopolítica neoliberal, pues esta falta de compromiso naturaliza, reproduce y asume de hecho el papel de las mujeres como cuidadoras. Por otra parte, a pesar de que el texto dedica un conjunto de medidas a ofrecer servicios que faciliten la conciliación, 
las mismas se basan en eludir la implicación de la Administración en la creación de servicios públicos, impulsando la creación de servicios privados o proporcionados por entidades sin ánimo de lucro. Este planteamiento, en consonancia con los objetivos de adelgazar el Estado y potenciar el mercado, va en detrimento de las familias más pobres, con más dificultades para la conciliación pero que no pueden acudir a los servicios del mercado para afrontarlas.

La parentalidad positiva se presenta en el PIAF 2015-2017 como un objetivo estratégico diferenciado. "El apoyo a padres y madres en el ejercicio de sus responsabilidades parentales, atendiendo al interés superior de los menores, constituye un objetivo básico de las políticas de apoyo a la familia" (p. 60). De hecho, es la otra cara de la moneda de la conciliación, la que intenta potenciar la implicación de padres y madres en la crianza, educación y cuidado de los hijos e hijas. La parentalidad positiva ha sido objeto de una Recomendación del Consejo de Europa (2006), que considera que las políticas públicas deben asumir un papel activo en la parentalidad, concediéndole más importancia y apoyo. La Recomendación surge de la constatación de que muchas familias consideran que no pueden incidir lo suficiente en el crecimiento y educación de sus hijos e hijas, por lo que buscan asesoramiento en su entorno familiar o de amistad y/o en los profesionales.

Los objetivos de la parentalidad positiva son coincidentes con la importancia que el PP otorga a la familia y la necesidad de potenciar sus funciones educativas y asistenciales. En este sentido, el PIAF 2015-2017 introduce numerosas medidas para potenciar la vida familiar y evitar algunos problemas de las familias, como la sensibilización hacia la parentalidad positiva y otras medidas cuyo principal objetivo son las dimensiones educativas, muchas de ellas vinculadas a la inclusión social.

\section{Menos Estado, más mercado, más familia}

Los objetivos políticos del PIAF 2015-2017 están claramente especificados en la introducción del documento, donde se señala la "necesidad de compatibilizar la política familiar con los objetivos de la política social y económica del Estado y con la viabilidad financiera de las distintas políticas de bienestar" (p. 4). Los énfasis que constantemente se hacen en el documento respecto a la función de la familia como principal amortiguador de las situaciones de dificultad económica y social, y respecto a sus responsabilidades en la atención de sus miembros hacen que se afirme que "esta realidad debe tenerse en cuenta en la elaboración y desarrollo de todas las políticas sociales" (p. 9), situando claramente al Estado en una posición complementaria, de apoyo y no de sustitución aunque sea parcial de estas funciones. El lenguaje político ensalza así la institución familiar, frente a un retraimiento del Estado en sus responsabilidades, congruente con lo que se denomina "viabilidad financiera" de las políticas de bienestar y que en los últimos años se ha traducido en planes de ajustes y recortes presupuestarios. Dicho claramente: más familia y menos Estado, más servicios privados y menos implicación pública.

Muchas de las 228 medidas que incluye el PIAF 2015-2017 contemplan la creación o consolidación de servicios. Es destacable que los servicios públicos son los más 
escasos y tratan de atender aspectos meramente asistenciales. Los programas de ayuda a los sectores más necesitados o de urgencia social se dejan en manos del Tercer Sector Social y el texto definitivo del PIAF incluso incrementa esta asignación respecto a los borradores anteriores. El resto de servicios se atribuye al sector privado, con la clara voluntad de protegerlo y ampliarlo, y favoreciéndolo mediante los cheques-servicios.

Este retraimiento de la implicación pública en favor de los servicios privados se muestra muy claramente comparando el PIAF 2015-2017 con los documentos elaborados desde distintos organismos e instituciones en los que se inspira. Uno de ellos es el Dictamen del Comité Económico y Social Europeo (2011) sobre política familiar y cambio demográfico. Una parte importante de las recomendaciones de este dictamen se recoge en el PIAF, y muchas de ellas incluso se reproducen literalmente. Sin embargo, cuando en el dictamen se habla de servicios públicos, en el PIAF se elimina sistemáticamente el término "público".

En el documento no aparecen discursos explícitos sobre las ventajas de recurrir a entidades privadas para la prestación de servicios. Se incorpora como algo dado, que no requiere mayor explicación ni justificación. De hecho, el fortalecimiento del sector privado y la colaboración público-privada se hallan presentes como objetivos en el Programa Nacional de Reformas del Reino de España, que anualmente presenta el gobierno a la Comisión Europea.

Esta inhibición del Estado frente a la potenciación del mercado tiene un marcado sesgo clasista, puesto que las capas sociales más pobres no pueden acceder a los servicios mercantiles. Para estos sectores, se reservan los servicios meramente asistenciales o la caridad organizada mediante la entrega de alimentos y el apoyo económico a las entidades que atienden las situaciones de pobreza más severa (PIAF 2015-2017, p. 34). Otro indicador del sesgo clasista se halla en las numerosas medidas fiscales, que permiten hacer deducciones del Impuesto sobre la Renta de las Personas Físicas (IRPF), lo que favorece claramente a las clases medias: los más pobres no pueden efectuar estas deducciones porque no alcanzan los ingresos mínimos para realizar las declaraciones pertinentes.

La reducción de la implicación del Estado en la creación de servicios incrementa a su vez la presión sobre las familias y sobre las mujeres, entrando nuevamente en clara contradicción con los principios de igualdad y corresponsabilidad. El PIAF no plantea por ejemplo, la recuperación de las medidas iniciales de la Ley de Dependencia, afectada por los recortes presupuestarios, por la supresión de la cotización de la seguridad social de las cuidadoras informales, o por el retraso en su aplicación. Como contrapunto, el PIAF sí ensalza constantemente la solidaridad intrageneracional e intergeneracional. Menos Estado supone pues más familia, más presión sobre las mujeres y retrocesos en la agenda de la igualdad. Se parte de la confianza de que las mujeres resolverán las situaciones de necesidad de cuidados, lo que es una expresión de unos dispositivos de poder que se asientan en la naturalización de los roles de género.

\section{Conclusiones}

El análisis del PIAF 2015-2017, en tanto que texto programático que debe guiar las políticas públicas en relación a la familia, permite no solo, como se decía al inicio de 
este texto, dilucidar cuál es el modelo de familia y de sociedad del gobierno que los ha promovido, sino también desvelar los mecanismos por los que la biopolítica trata de alcanzarlos. De hecho, el PIAF representa el camino inverso respecto a las políticas sociales que el gobierno del PSOE había arbitrado en los últimos años, orientadas a que la Administración hiciese frente a las situaciones de necesidad frente a los principios tradicionales del parentesco, de manera que las responsabilidades y obligaciones privadas de los familiares tendieran a ser sustituidas por la responsabilidad pública del Estado. Este enfoque de las políticas públicas no pretendía proteger a la familia, sino garantizar derechos individuales y proteger en todo caso a las personas con responsabilidades familiares. El PIAF 2015-2017 plantea las políticas públicas en dirección opuesta a esta: trata de fortalecer a la familia para fortalecer las responsabilidades de sus miembros. La idea de potenciar una familia centrada en la solidaridad intrageneracional e intergeneracional y de realzar su papel en la cohesión social por encima de los vínculos afectivos y emocionales entre sus miembros, denota una concepción tradicional de la institución y, sobre todo, una falta de voluntad para desarrollar políticas sociales. Aunque este tipo de relaciones familiares puede corresponder a determinados sectores de la sociedad, excluye a la gran diversidad de familias existentes hoy, a la importancia de los vínculos afectivos en el mantenimiento de las relaciones conyugales y, además, obvia que la carga de la obligación familiar recae especialmente en las mujeres.

El discurso político del texto establece jerarquías entre grupos familiares, exaltando y naturalizando un modelo determinado de familia -y de mujer y de maternidad- en detrimento de otras formas familiares, que son presentadas como "anomalías" del modelo preferente. La insistencia en la responsabilidad de la familia como eje vertebrador de la sociedad, unida a la escasez o ausencia de referencias explícitas tanto a los problemas actuales derivados de la crisis económica que está afectando - con frecuencia de forma dramática- a buena parte de las familias españolas como a las condiciones desiguales en que mujeres y hombres desempeñan sus funciones familiares puede leerse como una forma de "violencia eufemística" (Anagnost, 2000), que silencia los factores sociales y contextuales que contribuyen a las desigualdades entre grupos y géneros, como si estas fueran resultado de decisiones u opciones individuales.

\section{Documentos}

"Plan Integral de Apoyo a la Familia 2001-2004", Revista del Ministerio de Trabajo y Asuntos Sociales, 40: 105-124.

Plan Integral de Apoyo a la Familia. Propuestas de Objetivos, principios y líneas estratégicas. 14 de abril 2014.

Plan Integral de Apoyo a la Familia. 2014-2017. Borrador de trabajo. 7 de noviembre de 2014. Gobierno de España.

Plan Integral de Apoyo a la Familia, 2015-2017. Aprobado por Acuerdo del Consejo de Ministros de 14 de mayo de 2015. Gobierno de España. Disponible en web: http://www.msssi.gob.es/novedades/docs/PIAF-2015-2017.pdf [Consulta: 20 de abril de 2016] 


\section{Bibliografía}

Adsera, A. (2011): "Where Are the Babies? Labor Market Conditions and Fertility in Europe", European Journal of Population, 27(1), pp. 1-32. Disponible en: http://dx.doi.org/10.1007/s10680-010-9222-x.

Alberdi, I. (1997), "La familia. Convergencia y divergencia de los modelos familiares españoles en el entorno europeo", Política y Sociedad, 26, pp. 73-94.

Alvarez, B. (2013): "La maternidad: entre la decisión individual y/o la obligatoriedad social", en C. López, D. Marre y J. Bestard, (eds.), Maternidades, procreación y crianza en transformación, Bellaterra. Barcelona, Edicions Bellaterra.

Anagnost, A. (2000): "Scenes de Misrecognition: Maternal Citizenship in the Age of Transnational Adoption", Positions: East Asian Culture Critique, 8(2), pp. 389-421. Disponible en: http://dx.doi.org/10.1215/10679847-8-2-389

Arriba, A. y F.J. Moreno (2015), "A new legal framework to support families in Spain", European Social Policy Netwok - Flash report, European Comission.

Apthorpe, R. (1997): "Writing development policy and policy analysis plain or clear: On Language, gender and power", en C. Shore y S. Wright (eds.), Anthropology of Policy: Critical Perspectives of Governance and Power, London, Rouledge.

Bernardi, F. (2005): "Public policies and low fertility: rationales for public intervention and a diagnosis for the Spanish case", Journal of European Social Policy, 15(2), pp. Disponible en: 123-138. http://dx.doi.org/10.1177/0958928705049160.

Briggs, L. (2012): "Feminism and Transnational Adoption: Poverty, Precarity, and the Politics of Raising (Other People's) Children", Feminist Theory 13(1), pp. 81-100. Disponible en: http://dx.doi.org/10.1177/1464700111430177.

Bustelo, M. (2006): "Marcos interpretativos de las políticas de igualdad en Europa: conciliación, violencia y desigualdad de género en la política", Revista Española de Ciencia Política, 14, pp. 117-140.

Carrasco, C., C. Borderías y T. Torns, eds. (2011): El trabajo de cuidados: Historia, teoría y políticas, Madrid, La Catarata.

Carrasquer, P., M. Massó y A. Martín (2007): "Discursos y estrategias en torno a la conciliación de la vida familiar y laboral en la negociación colectiva", Papers, 83, 13-36.

Comas d'Argemir, D. (2012): "Políticas públicas y vida cotidiana: Redescubrimiento y auge de las políticas familiares en España", Scripta Nova. Revista electrónica de Geografía y Ciencias Sociales, vol. XVI, 395 (20). Disponible em: http://www.ub.es/geocrit/sn/sn-395/sn-395-20.htm.

Comas d'Argemir, D. (2014): "Los cuidados y sus máscaras. Retos para la antropología feminista", Mora (Buenos Aires), 20(1).

Comas d'Argemir, D. (2015): "Los cuidados de larga duración y el cuarto pilar del sistema de bienestar", Revista de Antropología Social, 24, pp. 375-404isponible en: http://dx.doi.org/10.5209/rev_RASO.2015.v24.50663 
Comisión Europea (2013), Recomendación: Invertir en la infancia: romper el ciclo de las desventajas, Diario Oficial de la Unión Europea 2.3.2013.

Comité Económico y Social Europeo (2011), Dictamen: El papel de la política familiar en el cambio demográfico: compartir las mejores prácticas entre los Estados miembros, Diario Oficial de la Unión Europea 23.7.2011.

Commaille, J. y C. Martin (1998): Les enjeux polítiques de la famille, París, Bayard.

Consejo General del Poder Judicial (2013): "Una aproximación a la conciliación de los datos sobre ejecuciones hipotecarias y deshaucios", Boletín Información Estadística, 35: $1-9$.

Dean, M. (1999): Governmentality: Power and Rule in Modern Society, London, Sage Publications.

Donzelot, J. ([1977] 1979): La policía de las familias, Valencia, Pre-textos.

Escot, L. y J.A. Fernández (coord.): Una evaluación de la introducción del permiso de paternidad de 13 días. ¿Ha fomentado una mayor corresponsabilidad en el ámbito del cuidado de los hijos pequeños?, Informe. Universidad Complutense de Madrid, Instituto de la Mujer, Fondo Social Europeo.

Fineman, M.A. (2000): "Cracking the Foundational Myths: Independence, Autonomy, and Self-Sufficiency", Journal of Gender, Social Policy and the Law, 8, pp. 13-29.

Flaquer, Ll. (2004a): "Llei de suport a les famílies. Una oportunitat perduda?", Working Paper de Dret Català, 12, Barcelona.

Flaquer, L1. (2004b): "La articulación entre família y el Estado del bienestar en los paises de la Europa del sur", Papers, 73, pp. 23-78.

Fonseca, C., D. Marre y B. San Román (2015): "Child Circulation in a globalized era: anthropological reflections", en R. L. Ballard, N. H. Goodno, R. F. Cochran y J. A. Milbrandt (eds.), The Intercountry Adoption Debate: Dialogues Across Disciplines, Newcastle upon Tyne, UK, Cambridge Scholars Publishing.

Foucault, M. ([1976] 2005): Historia de la sexualidad I. La voluntat de saber, Barcelona, Gedisa.

Gasper, L.D. y R. Apthorpe (1996): "Discourse analysis and policy discourse", en L. Apthorpe y L.D. Gasper (eds.), Arguing Development Policy. Frames and Discourses, London, Cass.

Gledhill, J. (1999): El poder y sus disfraces. Perspectivas antropológicas de la política, Barcelona, Bellaterra.

Instituto Nacional de Estadística (2001): “Cuántos somos en casa?”, Cifras INE. Boletín Informativo del Instituto Nacional de Estadística.

Instituto Nacional de Estadística (2011): Proyecto de los Censos Demográficos 2011.

Instituto Nacional de Estadística (2014): "Las formas de convivencia", Cifras INE. Boletín Informativo del Instituto Nacional de Estadística.

Kohler H.P., F.C. Billari y J.A. Ortega (2002): "The emergence of lowest-low fertility in Europe during the 1990s", Population and Development Review, 28, pp. 641-80. Disponible en:

http://dx.doi.org/10.1111/j.1728-4457.2002.00641.x. 
Krause E. y S. de Zordo, (2012): "Introduction. Ethnography and biopolitics: tracing 'rationalities' of reproduction across the north-south divide", Anthropology \& Medicine, 19(2), pp. 137-151. Disponible en: http://dx.doi.org/10.1080/13648470.2012.675050.

Lemke, T. (2001): 'The birth of bio-politics': Michel Foucault's lecture at the Collège de France on neo-liberal governmentality", Economy and Society, 30(2), 190-207. Disponible en: http://dx.doi.org/10.1080/03085140120042271

Lombardo, E. y M. León (2014): "Políticas de igualdad de género y sociales en España: origen, desarrollo y desmantelamiento en un contexto de crisis económica", Investigaciones Feministas, 15: 13-35. Disponible en: http://dx.doi.org/10.5209/ rev_INFE.2014.v5.47986

Marchesi, M. (2012): "Reproducing Italians: contested biopolitics in the age of 'replacement anxiety" “ Anthropology \& Medicine, 19(2), pp. 171-188. Disponible en: http://dx.doi.org/10.1080/13648470.2012.675043.

Marre, D. (2009): "Los silencios de la adopción en España", Revista de Antropología Social, 19, pp. 97-126.

Marre, D. (2011): "Cambios en la cultura de la adopción y de la filiación", en F. Chacón y J. Bestard, dirs., Familias. Historia de la sociedad española (del final de la Edad Media a nuestros días), Madrid, Cátedra.

Marre, D. y B. San Román (2012): “El 'interés superior' de la niñez en la adopción en España: entre la protección, los derechos y las interpretaciones", Scripta Nova, Revista electrónica de Geografia y Ciencias Sociales [En línea], vol. XVI, no 395 (9). Disponible en: http://www.ub.es/geocrit/sn/sn-395/sn-395-9.htm

Mishtal, J. (2014): "Reproductive Governance in the New Europe. Competing Visions of Morality, Sovereignty and Supranational Policy", Anthropological Journal of European Culture, 23(1), pp. 59-76. Disponible en: http://dx.doi.org/10.3167/ajec.2014.230104.

Morgan, S. P. y M. G. Taylor (2006): "Low Fertility at the Turn of the Twenty-First Century", Annual Review of Sociology, 32, pp. 375-99. Disponible en: http://dx.doi.org/10.1146/annurev.soc.31.041304.122220.

Ojakangas, M. (2007): “Impossible Dialogue on Bio-Power", Foucault Studies, 2, pp. 5-28.

Paleo, N. y A. Alonso (2014): “¿Es solo cuestión de austeridad? Crisis económica y políticas de género en España”, Investigaciones Feministas, 5, pp. 36-68. Disponible en:

http://dx.doi.org/10.5209/rev_INFE.2014.v5.47987

Pazos, M. (2013): Desiguales por ley. Las políticas públicas contra la igualdad de género, Madrid, Catarata.

Recio, C. (2015): "Políticas de conciliación de la vida familiar y laboral en España: una historia repleta de sombras", Revista de Estudios y Cultura, 69, pp. 8-12.

Rodríguez Cabrero, G. (2014): Invertir en la infancia: romper el ciclo de las desventajas. Un estudio sobre politicas nacionales. Informe nacional-España, Unión Europea. 
Ruiz-Huerta, J. (dir.) (2013): 1er informe sobre la desigualdad en España 2013, Madrid, Fundación Alternativas.

Salido, O. y L. Moreno (2007): "Bienestar y políticas familiares en España”, Política y Sociedad, 44, pp. 101-114.

Shore, C. y S. Wright (1997): "Policy, a new field on anthropology", en C. Shore y S. Wright, eds., Anthropology of Policy. Critical Perspectives of Governance and Power, Londres, Routledge.

Subirats, M. (1998): Con diferencia. Las mujeres frente al reto de la autonomía, Barcelona, Icaria.

Torns, T. (2005): "De la imposible conciliación a los permanentes malos arreglos", Cuadernos de Relaciones Laborales, 23, pp. 15-33.

UNICEF (2013): Bienestar infantil en los paises ricos. Un panorama comparativo. Florencia, UNICEF.

Valiente, C. (1996): "Olvidando el pasado: la política familiar en España (1975-1996)", Gestión y Análisis de Políticas Públicas, 5-6, pp. 151-162.

Valiente, C. (2013): "Gender equality policymaking in Spain: Losing momentum", en B.N. Field y A. Botti, eds. Politics and society in contemporary Spain. From Zapatero to Rajoy, Londres, Palgrave Macmillan.

Van Dijk, T. (2000): El discurso como interacción social, Barcelona, Gedisa. 